University of Nebraska - Lincoln

DigitalCommons@University of Nebraska - Lincoln

10-1-2005

Structures and stability of medium-sized silicon clusters. III. Reexamination of motif transition in growth pattern from $\mathrm{Si}_{15}$ to $\mathrm{Si}_{20}$

Soohaeng Yoo

University of Nebraska-Lincoln

Xiao Cheng Zeng

University of Nebraska-Lincoln, xzeng1@unl.edu

Follow this and additional works at: https://digitalcommons.unl.edu/chemzeng

Part of the Chemistry Commons

Yoo, Soohaeng and Zeng, Xiao Cheng, "Structures and stability of medium-sized silicon clusters. III. Reexamination of motif transition in growth pattern from $\mathrm{Si}_{15}$ to $\mathrm{Si}_{20}$ " (2005). Xiao Cheng Zeng Publications. 14.

https://digitalcommons.unl.edu/chemzeng/14

This Article is brought to you for free and open access by the Published Research - Department of Chemistry at DigitalCommons@University of Nebraska - Lincoln. It has been accepted for inclusion in Xiao Cheng Zeng Publications by an authorized administrator of DigitalCommons@University of Nebraska - Lincoln. 


\title{
Structures and stability of medium-sized silicon clusters. III. Reexamination of motif transition in growth pattern from $\mathrm{Si}_{15}$ to $\mathbf{S i}_{20}$
}

\author{
Soohaeng Yoo and X. C. Zeng ${ }^{\text {a) }}$ \\ Department of Chemistry and Center for Materials Research and Analysis, University of Nebraska-Lincoln, \\ Lincoln, Nebraska 68588
}

(Received 18 July 2005; accepted 3 August 2005; published online 24 October 2005)

\begin{abstract}
It has been established from experiments that stable medium-sized ionic clusters $\mathrm{Si}_{15}-\mathrm{Si}_{20}$ are prolate in shape. Density-functional theories (DFTs) also predict that nearly all low-lying neutral clusters in this size range are prolate in shape. Moreover, most of them are built onto two generic structural motifs, either the tricapped-trigonal-prism (TTP) $\mathrm{Si}_{9}$ motif or the six/six $\mathrm{Si}_{6} / \mathrm{Si}_{6}$ (sixfold-puckered hexagonal ring $\mathrm{Si}_{6}$ plus six-atom tetragonal bipyramid $\mathrm{Si}_{6}$ ) motif. However, it appears that the exact location of the TTP-to-six/six motif transition is dependent on the functional (e.g., PBE or BLYP) used in the DFT calculations. Here, we present total-energy calculations for two series of clusters (one series containing six/six motif and the other containing the TTP motif) in the size range of $\mathrm{Si}_{16}-\mathrm{Si}_{20}$. The calculations were based on all-electron DFT methods with a medium [6-311G $(2 d)]$ and a large (cc-pVTZ) basis sets, as well as coupled-cluster single and double substitutions (including triple excitations) [CCSD(T)] method with a modest (cc-pVDZ) basis set. In the DFT calculations, two popular hybrid density functionals, the B3LYP and PBE1PBE, were selected. It is found that the B3LYP total-energy calculations slightly favor the six/six motif, whereas the PBE1PBE calculations slightly favor the TTP motif. The CCSD(T) total-energy calculations, however, show that isomers based on the six/six motif are energetically slightly favorable in the size range of $\mathrm{Si}_{16}-\mathrm{Si}_{20}$. Hence, the TTP-to-six/six motif transition is more likely to occur at $\mathrm{Si}_{16}$. () 2005 American Institute of Physics. [DOI: 10.1063/1.2043127]
\end{abstract}

\section{INTRODUCTION}

In the previous two papers of this series (Papers I and II), ${ }^{1,2}$ we have presented $a b$ initio molecular-orbital calculations of geometric structures, total energies, vibrational frequencies, and ionization potentials of a number of smallsized $\left(\mathrm{Si}_{n}, n=7-11\right)$ and medium-sized clusters $\left(\mathrm{Si}_{n}, n\right.$ $=12-20)$. For medium-sized silicon clusters, most selected low-lying isomers were taken from those reported by Ho and co-workers ${ }^{3}$ who performed an unbiased search of globalminimum clusters with a combined tight-binding (TB)/ genetic algorithm method, as well as those by Rata et al. ${ }^{4}$ who performed an unbiased search with a density-functional TB method combined with single-parent evolution algorithm. Since publication of Paper II, a few new candidates of the global-minimum clusters in the size range $n=13-18$ have been reported in the literature, including $\mathrm{Si}_{13}$ and $\mathrm{Si}_{14}$ by Tekin and Hartke ${ }^{5}$ and $\mathrm{Si}_{16}-\mathrm{Si}_{18}$ in our previous work. ${ }^{6}$ In addition, we predicted that a TTP-to-six/six motif transition may occur at $\mathrm{Si}_{16}$. This prediction was based on a constrained (or biased) basin-hopping search with the six/six motif as the seeding, ${ }^{6}$ for clusters $\mathrm{Si}_{16}-\mathrm{Si}_{22}$. The search was coupled with the plane-wave-pseudopotential densityfunctional theory (PWP-DFT) with the Becke exchange and Lee-Yang-Parr correlation (BLYP) functional, implemented in the CPMD program. ${ }^{7}$ That search not only reproduced previously reported global-minimum structures of $\mathrm{Si}_{19}-\mathrm{Si}_{21}$ by Rata et $a l .,{ }^{4}$ but also resulted in new candidates for the global

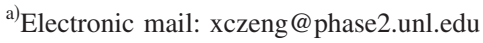

minima of $\mathrm{Si}_{16}-\mathrm{Si}_{18}$, and $\mathrm{Si}_{22}$. We showed that these new candidates built onto the six/six $\mathrm{Si}_{6} / \mathrm{Si}_{6}$ motif are lower in energy than the lowest-energy isomers reported previously. For $\mathrm{Si}_{16}$, we also performed an unconstrained basin-hopping search with the Perdew, Burke, and Ernzerhof (PBE) functional and obtained the same lowest-energy structure within limited basin-hopping Monte Carlo steps $(<5000)$.

Recently, Goedecker et al. ${ }^{8}$ reported a new candidate for the global minimum of $\mathrm{Si}_{16}$ as well as for $\mathrm{Si}_{19}$, based on a novel minima-hopping method ${ }^{9}$ and total-energy calculation using the PWP-DFT with the PBE functional implemented in the CPMD program. More interestingly, they found that these new structures of $\mathrm{Si}_{16}$ and $\mathrm{Si}_{19}$ all contain the TTP $\mathrm{Si}_{9}$ motif rather than the six/six $\mathrm{Si}_{6} / \mathrm{Si}_{6}$ motif. Because the total-energy differences calculated based on PWP-DFT/PBE method between the new candidates and previously reported ones are less than $0.01 \mathrm{eV} /$ at. (within the accuracy of PWP-DFT), there is a possibility that the obtained global-minimum structures are dependent on the functional selected. The purpose of this paper is to further examine this functional dependence by using all-electron DFT total-energy calculations associated with two popular hybrid functionals (implemented in GAUSSIAN 03 package ${ }^{10}$, which are Becke's three parameter hybrid functional with the Lee-Yang-Parr correlation $(B 3 L Y P)^{11}$ functional and the 1997 hybrid PBE $(\mathrm{PBE} 1 \mathrm{PBE})^{12}$ functional. We also examined this functional dependence on the predicted lowest-energy structures using two different basis sets: One medium basis set and one large one. Finally, we computed total energies for all candidate 
TABLE I. (A) Calculated total energies (in a. u.) and zero-point energies (ZPES) at various levels of theory for the six/six-motif-based clusters. (B) and (C) are the calculated energy differences (in eV) with respect to the corresponding six/six-motif-based isomers listed in (A). The bold-faced energy differences denote those isomers that have a lower energy than the corresponding isomers containing the six/six motif.

\begin{tabular}{|c|c|c|c|c|}
\hline \multirow{2}{*}{$\begin{array}{l}\text { (A) } \\
\text { Clusters }\end{array}$} & \multicolumn{2}{|c|}{ PBE1PBE/6-311G(2d) } & \multicolumn{2}{|c|}{ B3LYP/6-311G(2d) } \\
\hline & E (a.u.) & ZPE (a.u.) & E (a.u.) & ZPE (a.u.) \\
\hline $16 s s-1$ & -4630.1762501 & 0.028041 & -4632.2312719 & 0.026334 \\
\hline $17 \mathrm{ss}-1$ & -4919.5772322 & 0.030089 & -4921.7627080 & 0.028248 \\
\hline $18 \mathrm{ss}-1$ & -5208.9686517 & 0.031826 & -5211.2784797 & 0.029920 \\
\hline $19 \mathrm{ss}-1$ & -5498.3638781 & 0.032894 & -5500.7967827 & 0.030596 \\
\hline $20 \mathrm{ss}-1$ & -5787.7715689 & 0.035825 & -5790.3311343 & 0.033463 \\
\hline \multirow{2}{*}{ Clusters } & PBE1PBE/ & B3LYP/ & CCSD/cc-pVDZ & $\operatorname{CCSD}(\mathrm{T}) / \mathrm{cc}-\mathrm{pVDZ}$ \\
\hline & cc-pVTZ (a.u.) & cc-pVTZ (a.u.) & (a.u.) & (a.u.) \\
\hline 16 ss- 1 & -4630.2352858 & -4632.2777972 & -4624.3014583 & -4624.452846 \\
\hline $17 \mathrm{ss}-1$ & -4919.6401862 & -4921.8125448 & -4913.3438376 & -4913.5004829 \\
\hline $18 \mathrm{ss}-1$ & -5209.0349673 & -5211.3310528 & -5202.363120 & -5202.5322939 \\
\hline $19 \mathrm{ss}-1$ & -5498.4339710 & -5500.8516780 & -5491.3880334 & -5491.5742002 \\
\hline (B) & \multicolumn{2}{|c|}{ PBE1PBE/6-311(2d) } & \multicolumn{2}{|c|}{ B3LYP/6-311(2d) } \\
\hline Clusters & $\Delta E(\mathrm{eV})$ & $\Delta E+\Delta \mathrm{ZPE}(\mathrm{eV})$ & $\Delta E(\mathrm{eV})$ & $\Delta E+\Delta \mathrm{ZPE}(\mathrm{eV})$ \\
\hline 16bilayer-1 & 0.142 & 0.143 & 0.025 & 0.030 \\
\hline 16bilayer-1r & 0.122 & 0.107 & 0.258 & 0.238 \\
\hline 16cage-1 & 0.179 & 0.184 & 0.003 & 0.010 \\
\hline $16 \mathrm{ttp}-1$ & 0.187 & 0.152 & 0.655 & 0.607 \\
\hline $17 \mathrm{ttp}-1$ & 0.098 & 0.076 & 0.391 & 0.358 \\
\hline $18 \mathrm{ttp}-1$ & -0.333 & -0.338 & 0.195 & 0.174 \\
\hline $18 s s-2$ & -0.124 & -0.134 & 0.042 & 0.025 \\
\hline 19 ttp-1 & -0.033 & -0.030 & 0.419 & 0.412 \\
\hline 20 ttp-1 & 0.590 & 0.546 & 0.551 & 0.503 \\
\hline (C) & $\begin{array}{c}\text { PBE1PBE/cc-pVTZ } \\
(\mathrm{eV})\end{array}$ & $\begin{array}{c}\text { B3LYP/cc-pVTZ } \\
(\mathrm{eV})\end{array}$ & $\begin{array}{c}\text { CCSD/cc-pVDZ } \\
(\mathrm{eV})\end{array}$ & $\begin{array}{c}\operatorname{CCSD}(\mathrm{T}) / \mathrm{cc}-\mathrm{pVDZ} \\
(\mathrm{eV})\end{array}$ \\
\hline 16bilayer-1 & 0.109 & -0.005 & 0.116 & 0.042 \\
\hline 16bilayer-1r & 0.119 & 0.246 & 0.341 & 0.013 \\
\hline 16cage-1 & 0.124 & -0.022 & 0.116 & 0.004 \\
\hline $16 \mathrm{ttp}-1$ & 0.179 & 0.666 & 0.735 & 0.085 \\
\hline $17 \mathrm{ttp}-1$ & 0.082 & 0.393 & 0.582 & 0.080 \\
\hline $18 \mathrm{ttp}-1$ & -0.337 & 0.221 & 0.358 & 0.154 \\
\hline $18 s s-2$ & -0.127 & 0.055 & -0.90 & -0.125 \\
\hline 19 ttp-1 & -0.024 & 0.441 & 0.522 & 0.402 \\
\hline
\end{tabular}

clusters in the size range of $\mathrm{Si}_{16}-\mathrm{Si}_{19}$ using a high-level coupled-cluster theory with a modest basis set.

\section{COMPUTATIONAL DETAILS}

For all candidate clusters, we first performed geometric optimization using all-electron density-functional theory with both B3LYP and PBE1PBE hybrid functionals and the 6-311G $(2 d)$ (triple split for the valence basis functions plus polarization) basis set, compiled in the GAUSSIAN 03 software package. $^{10}$ Next, harmonic vibrational frequencies were computed using both DFT methods and the 6-311G $(2 d)$ basis set to assure that these clusters are local minima without any imaginary frequency. Zero-point energies (ZPES) were recorded and included in the total energies. Calculation re- sults are given in Table I. In order to examine the basis-set effects, we also performed geometric optimization using both B3LYP and PBE1PBE functionals and a larger Dunning's correlation consistent polarized valence triple zeta plus polarization (cc-pVTZ) basis set. ${ }^{13}$ Finally, single-point energies at the coupled-cluster single and double substitutions (including triple excitations) $[\mathrm{CCSD}(\mathrm{T})]$ level of theory with a modest Dunning's correlation consistent polarized valence double zeta plus polarization (cc-pVDZ) basis set were carried out on basis of the PBE1PBE/6-311G(2d) optimized geometries (Table I). $\operatorname{CCSD}(\mathrm{T})$ calculations based on B3LYP/6-311G(2d) optimized geometries were also performed, and the same energy orders were obtained as those based on PBE1PBE/6-311G(2d) optimized geometries. 


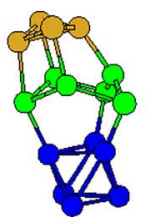

16ss-1 $\left(\mathrm{C}_{s}\right)$

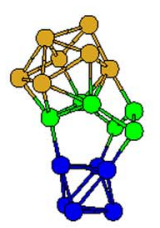

19ss-1 $\left(\mathrm{C}_{1}\right)$

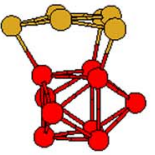

15ttp-1 $\left(\mathrm{C}_{3 v}\right)$

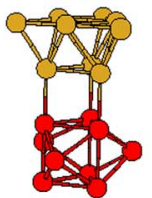

18ttp-1 $\left(\mathrm{C}_{s}\right)$

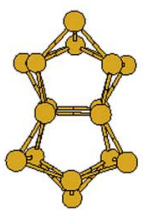

16bilayer-1

$\left(\mathrm{C}_{2 h}\right)$

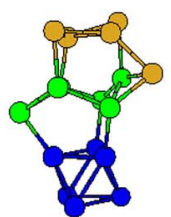

17ss-1 $\left(\mathrm{C}_{1}\right)$

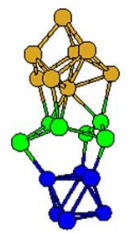

20ss-1 $\left(\mathrm{C}_{3 v}\right)$

(a)

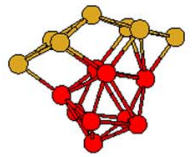

16ttp-1 $\left(\mathrm{C}_{s}\right)$

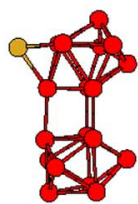

19ttp-1 $\left(\mathrm{C}_{s}\right)$

(b)

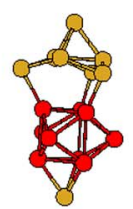

17ttp-1 $\left(\mathrm{C}_{3 v}\right)$

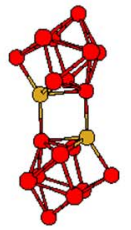

20ttp-1 $\left(\mathrm{C}_{2 h}\right)$
FIG. 1. (Color online). (a) Geometries of clusters with lowest (BLYP and B3LYP) energies and containing the six/six motif, i.e., the sixfold-puckered ring $\mathrm{Si}_{6}$ (green color) and the tetragonal bipyramid $\mathrm{Si}_{6}$ structural subunits (blue color). The yellow-colored atoms show growth patterns on the basis of the six/six motif. (b) Geometries of clusters with lowest (PBE and PBE1PBE) energies and containing the TTP motif, i.e., the tricapped-trigonal-prism $\mathrm{Si}_{9}$ motif (red color). (c) Geometries of other low-lying clusters of $\mathrm{Si}_{16}$ (Refs. 3 and 8) and $\mathrm{Si}_{18}$ (Ref. 4) predicted previously.

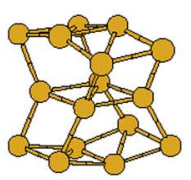

16bilayer-1r

$\left(\mathrm{C}_{1}\right)$

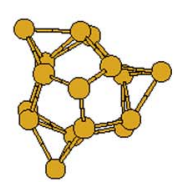

16cage-1

$\left(\mathrm{C}_{3}\right)$

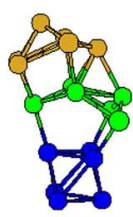

18ss-2

$\left(\mathrm{C}_{s}\right)$

(c)

\section{RESULTS AND DISCUSSIONS}

As pointed out in Sec. I, our attention will be given primarily to two series of low-lying clusters; one containing the six/six $\mathrm{Si}_{6} / \mathrm{Si}_{6}$ motif and another containing the TTP $\mathrm{Si}_{9}$ motif both in the size range of $\mathrm{Si}_{16}-\mathrm{Si}_{20}$. Figure 1(a) displays the lowest-energy isomers of the six/six-motif-based family, obtained previously based on the constrained (biased) basinhopping search coupled with PWP-DFT calculation with the BLYP functional. ${ }^{6}$ Hereafter, we name these six/six-motifbased lowest-energy isomers 16ss-1 to 20ss-1. In Fig. 1(a), the sixfold-puckered ring $\mathrm{Si}_{6}$ and the tetragonal bipyramid

$\mathrm{Si}_{6}$ subunits of the six/six motif are highlighted by the green and blue colors, respectively, whereas the yellow-colored atoms show growth patterns of these clusters on top of the six/six motif. Figure 1(b) displays the lowest-energy isomers containing the TTP (tricapped-trigonal-prism $\mathrm{Si}_{9}$ ) motif ${ }^{3}$ (highlighted in red color). Hereafter, we name these TTP-motif-based lowest-energy isomers $15 \mathrm{ttp}-1$ to $20 \mathrm{ttp}-1$. These isomers can be obtained based on a constrained basinhopping search with the TTP motif as the seeding. ${ }^{6}$ We performed this search in conjunction with PWP-DFT/PBE method (implemented in the CPMD program). For $\mathrm{Si}_{16}$ and 
$\mathrm{Si}_{19}$, we reproduced identical lowest-energy structures (16ttp-1 with $C_{s}$ symmetry and 19 ttp- 1 with $C_{s}$ symmetry) as reported by Goedecker et al., ${ }^{8}$ whereas for $\mathrm{Si}_{17}$ and $\mathrm{Si}_{18}$, we reproduced identical lowest-energy structures (17ttp-1 with $C_{3 v}$ symmetry and $18 \mathrm{ttp}-1$ with $C_{s}$ symmetry) reported by Ho et al. ${ }^{3}$ and our previous work, ${ }^{2}$ respectively. Interestingly, 19ttp-1 has the same geometric structure as the lowestenergy isomer of $\mathrm{Ge}_{19}$ predicted previously. ${ }^{14}$

In Table $\mathrm{I}(\mathrm{A})$, the total energies of the first series of isomers which contain the six/six motif are listed. The totalenergy differences of other isomers with respect to the corresponding six/six-motif-based isomers are given in Tables $\mathrm{I}(\mathrm{B})$ and $\mathrm{I}(\mathrm{C})$. These energy differences were calculated with various levels of theory and basis sets. Discussion of these results is given below for each size of clusters.

\section{A. $\mathrm{Si}_{16}$}

Goedecker et al. ${ }^{8}$ showed that $16 \mathrm{ttp}-1$ has the lowest energy whereas 16 ss-1 is the second in the energy order on the basis of PWP-DFT calculations with the PBE functional. However, on basis of all-electron DFT calculations with both B3LYP and PBE1PBE hybrid functionals and the 6-311G $(2 d)$ basis set, we found that 16 ss-1 is always lower in energy than 16ttp-1. The total-energy difference (including $\mathrm{ZPE})$ is $0.607 \mathrm{eV}$ (B3LYP) and $0.152 \mathrm{eV}$ (PBE1PBE), respectively. With the larger basis set (cc-pVTZ), the energy difference becomes slightly larger, i.e. 0.666 and $0.179 \mathrm{eV}$, respectively. The larger energy difference with the B3LYP functional appears to be consistent with our previous PWP-DFT calculations with the BLYP functional. As such, it seems that both B3LYP and BLYP functionals slightly favor the six/six-motif-based isomers over the TTP-motif-based isomers. The $a b$ initio energy calculations at the CCSD and $\operatorname{CCSD}(\mathrm{T})$ levels of theory with the cc-pVDZ basis set also show that the $16 \mathrm{ss}-1$ is lower in energy than $16 \mathrm{ttp}-1$, although the energy difference is much smaller $(0.085 \mathrm{eV})$ at the highest-level $\operatorname{CCSD}(\mathrm{T})$ theory.

In Fig. 1(c), we display one bilayer like isomer of $\mathrm{Si}_{16}$ named 16bilayer-1 and its variant named 16bilayer-1r. The 16bilayer isomer was previously predicted to be the global minimum by Ho et al., ${ }^{3}$ and its structure can be viewed as a network of eight parallel silicon dimers. The 16bilayer-1r, which was reported by Goedecker et al. as the third lowestenergy isomer, ${ }^{8}$ can be constructed by rotating three pairs of silicon dimers of 16 bilayer- 1 by $90^{\circ}$ at the top. In addition, we display a cagelike isomer of $\mathrm{Si}_{16}$ named 16cage-1. The 16cage-1 was obtained from a lengthy unconstrained basinhopping search combined with PWP-DFT with the BLYP functional. Note that when the PBE functional was used we found that 16cage-1 has an appreciably higher energy than both $16 \mathrm{ss}-1$ and $16 \mathrm{ttp}-1$. Here, in the all-electron DFT calculations, when the smaller basis set [6-311G $(2 d)]$ is used both B3LYP and PBE1PBE calculations show that 16cage-1 is higher in energy than 16ss-1. However, when the larger basis set (cc-pVTZ) is used, as shown in Table $\mathrm{I}(\mathrm{C})$, the B3LYP calculations show that 16cage-1 has the lowest energy (lower than all other four $\mathrm{Si}_{16}$ isomers), whereas the PBE1PBE calculations show that 16-ss1 still has the lowest energy. Both DFT calculations with the larger basis set however show that 16ttp-1 has the highest energy among the five $\mathrm{Si}_{16}$ isomers, and so do the CCSD/cc-pVDZ and CCSD(T)/cc-pVDZ energy calculations. Finally, the CCSD(T)/cc-pVDZ energy calculations show that the three $\mathrm{Si}_{16}$ isomers 16ss-1, 16cage-1, and 16bilayer-1r are very close in total energy (within $0.013 \mathrm{eV}$ ). Thus, a large basis set is needed with the $\operatorname{CCSD}(\mathrm{T})$ level of theory to determine the true energy order among these three $\mathrm{Si}_{16}$ isomers.

\section{B. $\mathrm{Si}_{17}$}

As reported previously, ${ }^{6}$ both B3LYP/6-311G $(2 d)$ and PBE1PBE/6-311G(2d) calculations as well as the CCSD/cc - $p$ VDZ and CCSD(T)/cc-pVDZ single-point energy calculations show that $17 \mathrm{ss}-1$ is lower in energy than $17 \mathrm{ttp}-1$. Even with the larger cc-pVTZ basis set for geometry optimizations, both DFT calculations show little changes in energy differences [see Tables I(B) and I(C) ]. Again, the energy difference calculated based on the PBE1PBE/cc-pVTZ theory $(0.082 \mathrm{eV})$ is appreciably less than that based on the B3LYP/ cc-pVTZ theory $(0.393 \mathrm{eV})$, indicating again that the PBE1PBE functional slightly favors the TTP-motif-based isomers whereas B3LYP slightly favors six/six-motif-based isomers.

\section{C. $\mathrm{Si}_{18}$}

$\mathrm{Si}_{18}$ is an interesting case since the total-energy order predicted based on the B3LYP calculations is opposite to that predicted based on the PBE1PBE calculations, regardless of sizes of the basis set considered here. Furthermore, the PBE1PBE total-energy calculations are consistent with the PWP-DFT calculations with the PBE functional. Because the energy order is apparently functional dependent, if one performs unbiased global search combined with DFT, the predicted lowest-energy structure will be dependent on the functional selected. Indeed, we found that this was even the case when we performed constrained (biased) basin-hopping search (for which the six/six motif was used as the seeding ${ }^{6}$ ) coupled with PWP-DFT and with the PBE functional, instead of the BLYP functional. With the PBE functional, we obtained the 18ss-2 as the lowest-energy isomer [Fig. 1(c)] rather than $18 \mathrm{ss}-1$. Note that $18 \mathrm{ss}-2$ has been previously reported by Rata et al. ${ }^{4}$ and studied in Paper II. This sensitivity of the predicted lowest-energy structure to the functional selected demonstrates that there exist several low-lying isomers with nearly the same energy for $\mathrm{Si}_{18}$. Hence, in order to determine the true global minimum of $\mathrm{Si}_{18}$, first-principles theories with much higher accuracy in total-energy calculation than DFT are required. Possible choices include quantum Monte Carlo method ${ }^{15}$ or $\operatorname{CCSD}(\mathrm{T})$ level of theory with a large basis set, e.g., cc-pVTZ or correlation-consistent palarized valence quadruple zeta (cc-pVQZ). The latter calculations are not yet feasible with current computing resources. Here, the CCSD(T)/cc-pVDZ calculations may still offer some guidance in the prediction of energy order, that is, the 18ss-2 structure may have a better chance as the candidate for the global minimum of $\mathrm{Si}_{18}$ over $18 \mathrm{ss}-1$ (having the 
lowest B3LYP energy) and 18ttp-1 (having the lowest PBE1PBE energy). If so, it still shows that this six/six-motifbased isomer is energetically favorable over the TTP-motifbased isomer for $\mathrm{Si}_{18}$.

\section{D. $\mathrm{Si}_{19}$}

The 19ttp-1 contains two TTP motifs and can be constructed by adding one atom on top of 18ttp-1 structure [Fig. 1(b)]. Thus, it is expected that when the PBE functional is selected in search for global minimum of $\mathrm{Si}_{19} 19 \mathrm{ttp}-1$ will be slightly favored over the six/six-motif-based isomers. We found that this is also the case when the all-electron PBE1PBE/6-311G(2d) and PBE1PBE/cc-pVTZ methods were used (see Table I). However, the total-energy difference between 19 ttp- 1 and $19 \mathrm{ss}-1$ is less than $0.04 \mathrm{eV}$ with the PBE1PBE calculations whereas the energy difference is an order of magnitude larger $(>0.4 \mathrm{eV})$ than the B3LYP calculations as well as with the CCSD/cc-pVDZ and $\operatorname{CCSD}(T) / c c-p V D Z$ calculations. Hence, the chance for the six/six-motif-based isomer 19ss-1 to be the true global minimum is higher than $19 \mathrm{ttp}-1$.

\section{E. $\mathrm{Si}_{20}$}

For $\mathrm{Si}_{20}$, the global-minimum structure was predicted to be 20 ss-1 by Rata et al. ${ }^{4}$ for which the total energies were calculated based on all-electron DFT with both PBE and Perdew-Wang-Becke 88 (PWB) functionals. As mentioned above, the PBE functional tends to slightly favor the TTP-motif-based isomers over the six/six-motif-based isomers in total-energy calculations. For $\mathrm{Si}_{20}$, the fact that even the DFT/PBE calculations show that the six/six-motif-based isomer 20ss-1 is energetically favorable over the TTP-motifbased isomer 20ttp-1 (Ref. 15) indicates that the TTP-to-six/ six motif transition occurs definitely at a cluster size smaller than $\mathrm{Si}_{20}$. Indeed, as shown in Table I, all-electron DFT calculations with both PBE1PBE and B3LYP functionals also support this indication. Moreover, the calculated total-energy differences amount to more than $0.5 \mathrm{eV}$ with both functionals. These results are consistent with our previous $\operatorname{CCSD}(\mathrm{T})$ calculations ${ }^{2}$ with a smaller basis set $[6-31 \mathrm{G}(d)]$, with which the calculated energy difference also amounts to about $0.4 \mathrm{eV}$. We thus expect that $\operatorname{CCSD}(\mathrm{T})$ calculations with larger basis sets may still give the same energy order as the DFT.

On basis of above total-energy results and previous ones, it is tempting to draw a tentative rule of thumb, that is, if both the B3LYP and PBE1PBE total-energy calculations (with a large basis set) give the same energy order and if the energy difference calculated with one of the functionals is greater than typical error bar of DFT for total-energy calculation $(\sim 0.2 \mathrm{eV}$ or less $),{ }^{16}$ then the $\operatorname{CCSD}(\mathrm{T})$ energy order will be most likely the same as predicted by the DFT. Thus far, we have not seen any exception to this simple rule of thumb. If this rule is further tested and proved for other larger clusters, one may no longer needs to perform very expensive (or impractical) $\mathrm{CCSD}(\mathrm{T})$ total-energy calculations for large-sized silicon clusters. On the other hand, we note that if the B3LYP energy order is different from the
PBE1PBE energy order, as in the cases of $\mathrm{Si}_{16}$ and $\mathrm{Si}_{18}$, one has to perform high-level first-principles calculations [e.g., quantum Monte Carlo or CCSD(T) with a large basis set] to determine the true global-minimum structure.

\section{CONCLUSIONS}

We have performed total-energy calculations for prolateshaped low-lying silicon clusters ${ }^{17}$ in the size range of $\mathrm{Si}_{16}-\mathrm{Si}_{20}$ using all-electron DFT with two hybrid functionals B3LYP and PBE1PBE, and ab initio theory at the CCSD(T) level. Particular attention is placed on two series of lowlying clusters: one containing the TTP $\mathrm{Si}_{9}$ structural motif whereas another containing the six/six $\mathrm{Si}_{6} / \mathrm{Si}_{6}$ structural motif. It is found that the B3LYP total-energy calculations slightly favor the six/six-motif-based isomers whereas the PBE1PBE calculations slightly favor the TTP-motif-based isomers. Consequently, when the total-energy difference between the low-lying isomers is less than typical accuracy of total-energy calculations of DFT, the predicted global minimum can be dependent on the functional selected. This is particularly seen in the cases of $\mathrm{Si}_{18}$ and $\mathrm{Si}_{19}$. On the other hand, these DFT results still support that isomers containing either the TTP or the six/six structural motif are energetically very favorable in the size range $\mathrm{Si}_{16}-\mathrm{Si}_{19}$. Previous theoretical studies have shown that TTP structural motif is prevailing in the small-sized low-lying clusters of $\mathrm{Si}_{11}-\mathrm{Si}_{15}$ (Ref. 3), whereas the six/six or six/ten (the ten refers to magic-number cluster $\mathrm{Si}_{10}$ ) structural motif is energetically more favorable in the medium-sized low-lying clusters $\mathrm{Si}_{20}-\mathrm{Si}_{26}$ (Refs. 3, 6, and 18). So the TTP-to-six/six motif transition is likely to occur in the range of $\mathrm{Si}_{16}-\mathrm{Si}_{19}$.

Although the exact location for the motif transition cannot be determined unambiguously with DFT total-energy calculations, the observation of the two generic structural features is still of fundamental importance towards the understanding of the structural evolution of silicon clusters. It is known that as the size of cluster increases, locating the global minima becomes increasingly difficult because of the much increased complexity of the potential energy surface as well as the rapid increase of the number of low-lying isomers. Therefore, any identification of certain types of generic structural feature (e.g., TTP motif) can dramatically reduce computation cost for the first-principles global search and more importantly it can provide additional physical insight (or guide) into growth patterns of medium-to-large-sized clusters. For example, we have recently used the carbon fullerene as structural motifs ${ }^{19}$ to construct "stuffed fullerene-type" ${ }^{\text {20,21 }}$ low-lying medium-sized clusters $\mathrm{Si}_{40}, \mathrm{Si}_{45}$, and $\mathrm{Si}_{50}$. The physical insight for this fullerenecage-based cluster construction was gained through an unbiased global search using genetic algorithm combined with TB method. ${ }^{22}$

Finally, we note that the present $a b$ initio total-energy calculations at the $\operatorname{CCSD}(\mathrm{T})$ level with a modest basis set (cc-pVDZ) show that the six/six-motif-based isomer series generally has a lower energy than the corresponding TTP-motif-based isomer series for $\mathrm{Si}_{16}-\mathrm{Si}_{19}$. More definite determination of the relative energy between these two series 
requires much larger basis sets, e.g., cc-pVTZ or cc-pVQZ. Such a calculation is currently beyond our computing capability. Nevertheless, we have recently performed CCSD(T)/cc-pVQZ, CCSD(T)/cc-pVTZ, and $\operatorname{CCSD}(\mathrm{T}) / \mathrm{cc}-\mathrm{pVDZ}$ total-energy calculations ${ }^{23}$ for two nearly isoenergy isomers of $\mathrm{Si}_{6}$ with their energy difference on the order of $0.01 \mathrm{eV}$ [based on $\operatorname{CCSD}(\mathrm{T}) / \mathrm{cc}-\mathrm{pVQZ}$ calculations]. We found that the $\operatorname{CCSD}(\mathrm{T}) / \mathrm{cc}-\mathrm{pVDZ}$ calculations predicted the same energy order as $\operatorname{CCSD}(\mathrm{T}) / \mathrm{cc}-\mathrm{pVQZ}$ but underestimates the energy difference $(0.002 \mathrm{eV})$. Assuming the accuracy of total-energy calculations with $\operatorname{CCSD}(\mathrm{T}) / \mathrm{cc}-\mathrm{pVDZ}$ is on the order of $0.1 \mathrm{eV}$ for mediumsized silicon clusters, we then expect that the calculated energy differences $(0.28 \mathrm{eV})$ between $18 \mathrm{ss}-2$ and $18 \mathrm{ttp}-1$, and $(0.402 \mathrm{eV})$ between $19 \mathrm{ss}-1$ and $19 \mathrm{ttp}-1$ are fairly reliable. As such, $18 \mathrm{ss}-2$ and $19 \mathrm{ss}-1$ are very likely to be the global minimum compared to $18 \mathrm{ttp}-1$ and $19 \mathrm{ttp}-1$, respectively. In other words, the TTP-to-six/six motif transition is most likely to occur at a cluster size less than $\mathrm{Si}_{18}$.

\section{ACKNOWLEDGMENTS}

We are very grateful to valuable discussions with Professor Th. Frauenheim, Professor K.A. Jackson, and W. Hellmann. This research was supported by grants from DOE (Grant No. DE-FG02-04ER46164), NSF (Grant NOs. DMII0210850, CHE-0427746, and MRSEC), John Simon Guggenheim Foundation, the Nebraska Research Initiative, and by the research computing facility at the University of Nebraska-Lincoln.
${ }^{1}$ X. Zhu and X. C. Zeng, J. Chem. Phys. 118, 3558 (2003).

${ }^{2}$ X. L. Zhu, X. C. Zeng, Y. A. Lei, and B. Pan, J. Chem. Phys. 120, 8985 (2004).

${ }^{3}$ K.-M. Ho, A. A. Shvartsburg, B. Pan, Z.-Y. Lu, C.-Z. Wang, J. G. Wacker, J. L. Fye, and M. F. Jarrold, Nature (London) 392, 582 (1998); B. Liu, Z.-Y. Lu, B. Pan, C.-Z. Wang, K.-M. Ho, A. A. Shvartsburg, and M. F. Jarrold, J. Chem. Phys. 109, 9401 (1998).

${ }^{4}$ I. Rata, A. A. Shvartsburg, M. Horoi, Th. Frauenheim, K. W. M. Siu, and K. A. Jackson, Phys. Rev. Lett. 85, 546 (2000).

${ }^{5}$ A. Tekin and B. Hartke, Phys. Chem. Chem. Phys. 6, 503 (2004).

${ }^{6}$ S. Yoo and X. C. Zeng, Angew. Chem., Int. Ed. 44, 1491 (2005).

${ }^{7}$ J. Hutter, A. Alavi, T. Deutsch, M. Bernasconi, S. Goedecker, D. Marx, M. Tuckerman, and M. Parrinello, CPMD, Version 3.7.1, MPI für Festkörperforschung, Stuttgart, 1997.

${ }^{8}$ S. Goedecker, W. Hellmann, and T. Lenosky, Phys. Rev. Lett. 95, 055501 (2005).

${ }^{9}$ S. Goedecker, J. Chem. Phys. 120, 9911 (2004).

${ }^{10}$ M. J. Frisch, G. W. Trucks, H. B. Schlegel, et al., GaUSSIAN 03, Revision C.02,Gaussian, Inc., Wallingford CT, 2004.

${ }^{11}$ J. P. Perdew, Phys. Rev. B 33, 8822 (1986); A. D. Becke, Phys. Rev. A 38, 3098 (1988); C. Lee, W. Yang, and R. G. Parr, Phys. Rev. B 37, 785 (1988); A. D. Becke, J. Chem. Phys. 98, 5648 (1993).

${ }^{12}$ P. Perdew, K. Burke, and M. Ernzerhof, Phys. Rev. Lett. 77, 3865 (1996); J. P. Perdew, K. Burke, and M. Ernzerhof, Phys. Rev. Lett. 78, 1396 (1997)

${ }^{13}$ T. H. Dunning, J. Chem. Phys. 90, 1007 (1989).

${ }^{14}$ S. Bulusu, S. Yoo, and X. C. Zeng, J. Chem. Phys. 122, 164305 (2005).

${ }^{15}$ L. Mitas, J. C. Grossman, I. Stich, and J. Tobik, Phys. Rev. Lett. 84, 1479 (2000).

${ }^{16}$ D. Young, Computational Chemistry (Wiley, New York, 2001).

${ }^{17}$ M. F. Jarrold and V. A. Constant, Phys. Rev. Lett. 67, 2994 (1991)

${ }^{18}$ K. A. Jackson, M. Horoi, I. Chaudhuri, T. Frauenheim, and A. A. Shvartsburg, Phys. Rev. Lett. 93, 013401 (2004).

${ }^{19}$ J. Zhao, J. Wang, J. Jellinek, S. Yoo, and X. C. Zeng, Eur. Phys. J. D 34, 35 (2005)

${ }^{20}$ U. Rothlisberger, W. Andreoni, and M. Parrinello, Phys. Rev. Lett. 72, 665 (1994).

${ }^{21}$ Q. Sun, Q. Wang, P. Jena, S. Waterman, and Y. Kawazoe, Phys. Rev. A 67, $063201(2003)$

${ }^{22}$ S. Yoo, J. J. Zhao, J. L. Wang, and X. C. Zeng, J. Am. Chem. Soc. 126, 13845 (2004)

${ }^{23}$ Y. Gao, C. Killblane, and X. C. Zeng (unpublished). 